\title{
Perceptual latency priming and illusory line motion: Facilitation by gradients of attention?
}

\author{
Ingrid Scharlau and Gernot Horstmann
}

Department of Psychology, Bielefeld University, Bielefeld, Germany

Received 26.04.2005

Accepted 15.12.2005

\section{Keywords}

Priming, attention, metacontrast, perceptual latency

\begin{abstract}
The phenomena of illusory line motion and perceptual latency priming are both assumed to reflect a facilitation of perceptual latency. The explanation of illusory line motion presupposes that attention is distributed in a gradient fash-

ion whereas this is not a necessary part of the explanation of perceptual latency priming. Two experiments test whether an attentional gradient is present in perceptual latency priming. Evidence for a gradient was found within $2.5^{\circ}$ of visual angle around the attended location, but not at a distance of $5^{\circ}$ and more.
\end{abstract}

\section{PERCEPTUAL LATENCY PRIMING AND ILLUSORY LINE MOTION: FACILITATION BY GRADIENTS OF ATTENTION?}

It is commonly assumed that visuospatial attention facilitates the processing of visual information. Compared to an unattended stimulus, attended stimuli are processed more efficiently, elaborately, and accurately (e.g., Eriksen \& Hoffman, 1972; Hawkins, Hillyard, Luck, \& Mouloua, 1990; Müller \& Findlay, 1987; Posner, 1980; Treisman, 1988). Processing of attended information also proceeds faster than that of unattended events. This advantage leads to socalled prior entry: Faster processing decreases the perceptual latency of a stimulus. When an attended and an unattended stimulus are presented simultaneously, the attended one is perceived earlier, or as the earlier event. (The term 'prior entry' dates back to Wundt and Titchener who explained the phenomenon by claiming that that the attended stimulus entered consciousness prior to an unattended stimulus; see, e.g., Titchener, 1908; Wundt, 1887. Today, the moment of conscious perception is not a defining part of the explanation of prior entry.)
Prior entry has been investigated since the earliest days of experimental psychology, and in fact even before the rise of psychology (e.g., Bessel, 1838; Boring, 1929; Sanford, 1888; Wundt, 1887; see Scharlau, 2005, for more detail). In the last decades, two experimental paradigms have been established by means of which prior entry can be studied: illusory line motion and perceptual latency priming. Although these two phenomena are assumed to rely on the same functional basis, that is, prior entry, they have been jointly studied only once (Scharlau, 2004b), and their explanations are partly contradictory.

In perceptual latency priming (PLP), attention-mediated latency facilitation is caused by an invisible ( $v i-$ sually backward masked) peripheral cue, the so-called prime (e.g., Neumann, 1982; Neumann, Esselmann, \& Klotz, 1993; Scharlau \& Neumann, 2003a; Steglich \& Neumann, 2000). The prime appears at the location of one of two stimuli, and, as assessed by temporal

Inquiries or requests for reprints should be sent to: Ingrid Scharlau, Department of Psychology, Bielefeld University, P.O. Box 1001 31, D-33501 Bielefeld, Germany, Tel.: ++49 (0) 521106 4505, e-mail: ingrid.scharlau@uni-bielefeld.de, http://www.uni-bielefeld.de/psychologie/ae/Ae01/hp/scharlau 
order judgments, the primed stimulus is perceived as the earlier one if it is presented simultaneously with the other stimulus and even if it briefly trails the latter. Masking the prime is not necessary for PLP to occur (Shore, Spence, \& Klein, 2001; Stelmach, Campsall, \& Herdman, 1997; Stelmach \& Herdman, 1991; Zackon, Casson, Zafar, Stelmach, \& Racette, 1999). It is, however, a convenient means to reduce judgment biases, that is, to prevent the observers from judging the prime instead of the relevant stimuli (Scharlau, 2002, 2004a). For example, Shore et al. reported that a prior-entry effect of $64 \mathrm{~ms}$ with visible cues was accompanied by a substantial judgment bias of $16 \mathrm{~ms}$. In contrast, no significant judgment bias was found in an earlier study when the cue was masked (Scharlau, 2004a). Probably, the masked information is too weak to induce a judgment tendency.

In illusory line motion (ILM), attention-mediated latency facilitation is caused by a visible cue (e.g., Hikosaka, Miyauchi, \& Shimojo, 1993a, b, c). This cue precedes a stationary line at one of its ends. The impression is one of motion, unfolding from the attended end towards the unattended end. According to the attentional account of ILM, the cue captures attention, which facilitates processing and thus reduces the perceptual latency of the line parts by an amount proportional to the distance from the cue, that is, to the focus of attention (gradient account). Central motion detectors register motion because of the asynchrony with which codes representing the near and the far ends of the line reach these detectors (e.g., Shimojo, Hikosaka, \& Miyauchi, 1999). The explanation of PLP is very similar, except that it does not presuppose a gradient account of attention and does not rely on motion detection (Scharlau \& Neumann, 2003a).

Scharlau (2004b) recently compared motion judgments (ILM) and temporal-order judgments (PLP) within the same experiment and, importantly, under identical stimulus conditions. The amount of latency facilitation found with these two methods was identical. This finding supports in principle a close affinity, and a common attentional explanation, of both phenomena. However, earlier studies cast some doubts on this.

(1) In earlier experiments, we did not find unequivocal evidence for a gradient of attention. Indeed, Scharlau (2004c) found a graded distribution only when the two stimuli remained on the screen. Even then, statistical evidence for gradients was weak - although numerically, a gradient was present.
(2) The ILM experiments prove that motion is perceived, but they do not directly support the claim that motion results from a gradient of attention. Motion can, for instance, be due to classical apparent (stroboscopic) motion between cue and line (Downing \& Treisman, 1997).

(3) Most of the ILM experiments are open to response biases (Pashler, 1998). Observers have to indicate with a two-alternative judgment the direction of motion within the line. Suppose that they do not perceive motion (the line is in fact stationary). They would then have to rely on criteria other than motion for their judgments. One such criterion is the presence of a cue or the direction of attention. According to Pashler, observers may ascribe very different criteria or features to the cued location or stimulus - for example being the first one, the brighter one, or the last one. Being the starting point of motion could be such a criterion, or feature, too. As far as we know, the bias argument has not been studied empirically in the ILM paradigm. There are, however, several studies on response bias in PLP. Shore et al. (2001) showed that there was indeed a response bias when attention was attracted by a visible cue (and an even larger bias when attention was voluntarily directed towards a certain location). PLP by an invisible prime, by contrast, is free from response bias (Scharlau, 2004a).

The spatial distribution of attention is currently one of the major topics in cognitive psychology. ILM is one of the phenomena which have been used to support the gradient theory of attention, according to which attention is distributed gradedly around its focus, the strength of attentional facilitation decreasing with distance (e.g., Downing, 1988; Handy, Kingstone, \& Mangun, 1996; Henderson \& Macquistan, 1993; Hikosaka et al., 1993a, b, c; Mangun \& Hillyard, 1988; McCormick \& Klein, 1990). Though evidence for gradients of attention has been revealed in different paradigms, it is not unequivocal. First, some researchers did not find gradients (Goolkasian \& Tarantino, 1999; Hughes \& Zimba, 1985, 1987) or reported that gradients were contingent on the presence of landmarks in the visual field (Scharlau, 2004c; Zimba \& Hughes, 1987), on a spatially defined response (Hodgson, Müller, \& O'Leary, 1999) or on other task demands (Downing, 1988; Handy et al., 1996). Second, and as mentioned above, in most of the studies by Hikosaka and colleagues, gradients were inferred from motion, but there was no straightforward evidence for them, and neither the size nor the slope of the attentional gradient were reported. By contrast, von Grünau and coworkers have developed a method which allows one to estimate laten- 
cy facilitation as well as size and slope of the gradient: They combine stroboscopic motion, or a luminance gradient within the bar, with cue-induced ILM and report the temporal or spatial parameters where these types of motion cancel each other (von Grünau, Racette, \& Kwas, 1996; von Grünau, Saikali, \& Faubert, 1995). In this paradigm, response-time data provided the basis for inferring latency facilitation. Direct evidence for gradients was present, although it varied considerably in size and slope between individual observers, and one observer even showed a reversed gradient (von Grünau et al., 1996). Third, attentional gradients can have different forms (cf. Turatto \& Galfano, 2001) and only the typical form seems to be readily reconcilable with the perception of motion in a uniform direction.

Both the alternative explanations and the ambiguous evidence for gradients render an independent confirmation of attentional gradients in latency facilitation desirable. Such an opportunity is provided by PLP.

The rationale of the present study is that if an attentional gradient exists, stimuli should be facilitated by an amount proportional to their distance from the attentional focus. A stimulus that is presented at the prime's location - the attentional focus - receives maximum facilitation. A second stimulus is facilitated depending on its proximity to the focus - more if it is near and less if it is far from the focus. If this second stimulus receives facilitation as well, the relative headstart of the stimulus at the primed location is reduced, resulting in a smaller net PLP value.

In the following experiments, the observers judge the temporal order of two asynchronous visual sti-muli. Prior to the stimuli, attention is drawn towards the location of one stimulus by a masked prime. If gradients of attention arise, a distance effect is expected: PLP for the primed stimulus should increase with distance between the two stimuli. If the distance is small, the second stimulus may fall inside the gradient and likewise achieve some facilitation, which in turn reduces the latency advantage of the primed stimulus. If the distance is large, an advantage for the primed stimulus relative to the unprimed stimulus should be larger.

Besides priming and distance, we varied the presence of a line in the display which connected the two stimuli. Hamm and Klein (2002) reported that cueing one end of a line produced attention-mediated benefits at the other end, but not at a location that was equidistant but unconnected to the cue. Attentional gradients may thus require the presence of stimulation along their axis (see also Zimba \& Hughes, 1987).

\section{EXPERIMENT 1}

\section{Method}

\section{Participants}

Seven women and 3 men (mean age 26.2 years, normal or corrected-to-normal visual acuity) whose informed consent was obtained received $€ 6$ for participation.

\section{Apparatus, stimuli, and procedure}

The stimuli were presented on a light gray background on a color monitor (refresh rate $62 \mathrm{~Hz}$ ) in a dimly lit room. A chin rest fixed the viewing distance at $60 \mathrm{~cm}$. The observers responded by pressing o ne of the buttons of a computer mouse.

The observers reported the temporal order of two well visible, asynchronous targets, one with a red, and one with a blue center. These targets were $3 \times 3$ checkerboards with 4 dark gray squares at the corners, and 4 light gray squares at the sides. The center square was red or blue, which was the reported feature ("red first" or "blue first"). The targets' edges were $1.4^{\circ}$ of visual angle. In half of the trials, a prime consisting of a single dark gray square $\left(0.5^{\circ}\right)$ preceded one of the checkerboards. The prime was presented at the same location as the light gray square of the checkerboard that was closest to fixation (e.g., the right side if the checkerboard was on the left side of the screen). It was thus surrounded by two dark gray squares above and below, and a colored square either to the right or to the left. This arrangement met the conditions for metacontrast masking of the prime. (The prime might also be termed "masked cue" since its main function is to attract attention to its location.) The distance between centers of the targets was either $5.4^{\circ}$ or $9.1^{\circ}$. These distances were based on earlier studies on ILM ( $9^{\circ}$ in Hikosaka et al., 1993a; and $5^{\circ}$ in von Grünau et al., 1995, 1996). A distance of $5.4^{\circ}$ should be a within-gradient distance, while a $9.1^{\circ}$ distance should be at the edge of, or outside, the gradient. In half of the trials, the targets were connected by a line $0.5^{\circ}$ in width and $4.5^{\circ}$ or $8.2^{\circ}$ in length. The targets (and the line, if present) were presented either horizontally or vertically, the minimal distance from fixation being $2.7^{\circ}$. A black fixation cross was present throughout the experiment (see Figure 1 for the spatial layout).

Prime duration was $32 \mathrm{~ms}$; the other stimuli were turned off simultaneously with the judgment. Stimulusonset asynchronies (target SOAs) were $-64,-32,0$, +32 , and $+64 \mathrm{~ms}$. Negative numbers indicate that the primed target led the unprimed target; positive SOAs indicate that the unprimed target appeared first. In 

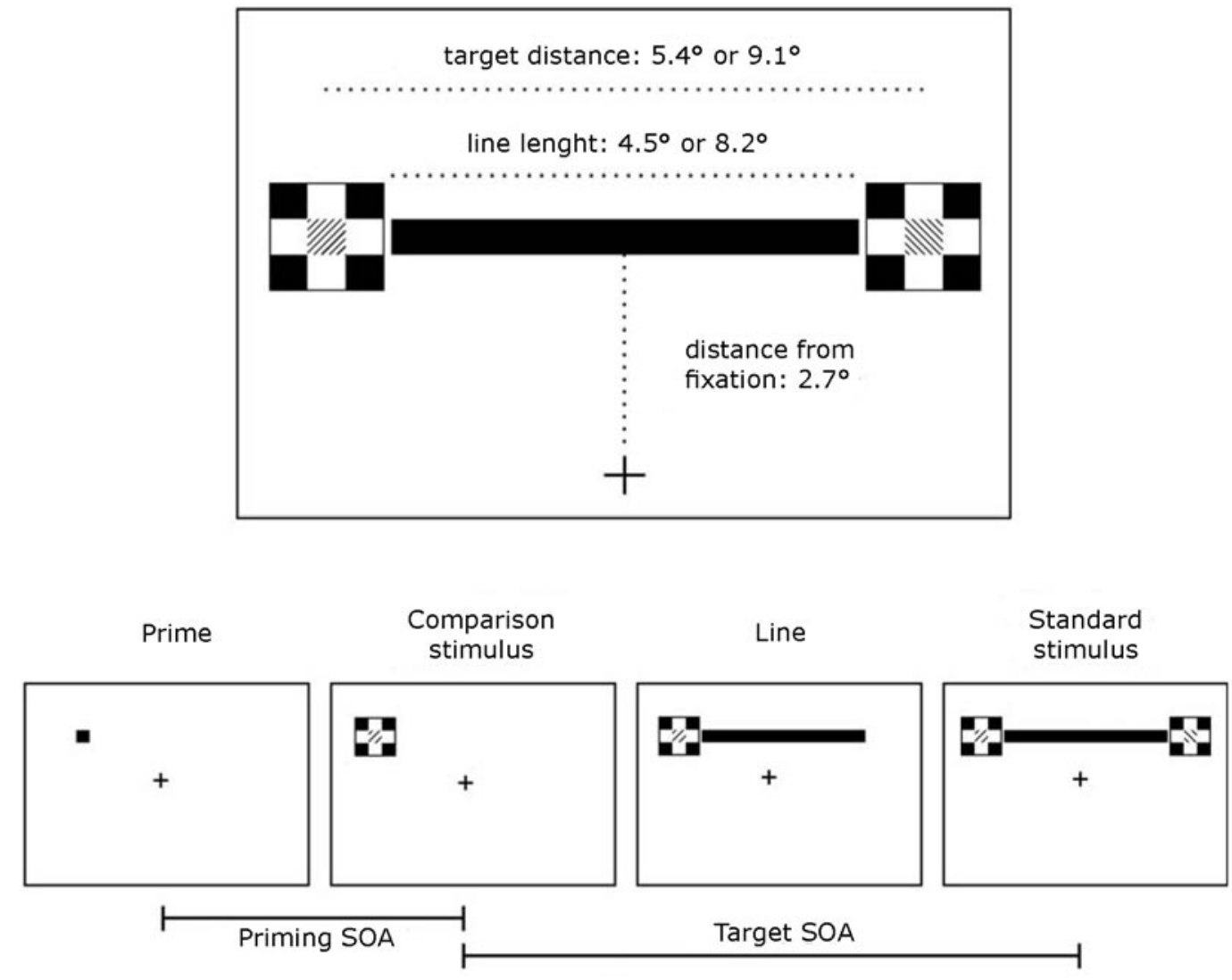

\section{Figure 1.}

Stimuli and spatial layout (top panel) and succession of events in a sample trial of Experiment 1 (bottom panel). Stimuli are not drawn to scale. Colors are indicated by patterns.

trials without a prime, positive and negative values were randomly assigned. Primes preceded the primed target by $96 \mathrm{~ms}$ (priming SOA). The line's onset was exactly intermediate to the onsets of the two targets.

There were 40 conditions ( 5 target SOAs $\times 2$ distance conditions $\times 2$ line conditions $\times 2$ priming conditions). Each condition was presented 32 times using the method of constant stimuli.

The participants judged the temporal order of the targets with a ternary judgment including two order categories ("red first" vs. "blue first") and a simultaneous/ doubt category ("simultaneous/unclear") (Ulrich, 1987). Half of the participants responded with the left mouse button if they saw the red target first, and with the right button if they perceived the blue target first. The assignment was reversed for the other half. The center button could be used to indicate simultaneity or uncertainty.

\section{Results}

The parameter of most interest is the point of subjective simultaneity (PSS), that is, the stimulus-onset asynchrony at which the two targets are perceived as simultaneous. If latency facilitation arises, the PSS should not coincide with actual simultaneity but with an SOA at which the primed stimulus is presented after the unprimed stimulus: Due to attention-mediated speed-up of processing, the primed target can make up for this lag. The PSS was calculated as the center of gravity of the approximated logistic function $\left(P=1 /\left\{1+e^{-(a+\beta x)}\right\}\right)$ (Finney, 1971); PSS is equivalent to the parameter a of the logistic regression. PSS calculation disposes of the factor SOA so that for the subsequent ANOVA three factors, each with two steps, are used (priming, that is, prime absent vs. prime present, distance, that is, $5.4^{\circ}$ vs. $9.1^{\circ}$, and line presence, that is, without line or with line).

A three-way ANOVA of PSS revealed a main effect of priming $[F(1,9)=51.18, p<.001]$. There were no main effects of distance or line presence (both Fs $<1, p s>.5$ ), and all interactions failed to reach significance (all $F s<1.58$, ps $>.24$ ). PSS values were $0 \mathrm{~ms}$ in the unprimed and $+40 \mathrm{~ms}$ in the primed condition. Figure 2 (top panel) depicts the PSS; per- 
ceptual latency priming is the difference between the PSS of unprimed and the PSS of primed conditions. However, the power of the statistical test to detect an influence of distance or an interaction between distance and line presence is rather small: It is .519, given an $a$-level of .25 for distance effects and interaction of priming and distance (Faul \& Erdfelder, 1992). That is, the probability that a distance effect is present but not statistically significant is rather large $(\beta=.481)$.

All PLP values differed significantly from Zero (all ts $\geq 3.43$, all $p s<.01)$, that is, latency facilitation or PLP was present in all of the primed conditions in Experiment 1.

\section{Discussion}

The order judgments revealed robust latency facilitation of approximately $40 \mathrm{~ms}$ with a priming interval of $96 \mathrm{~ms}$. However, the PSS results do not indicate that a gradient was present. Latency facilitation did not differ significantly for the $5.4^{\circ}$ and $9.1^{\circ}$ distances between the attention-attracting prime and the target at the unprimed location. That is, we found no statistical difference in attention-mediated facilitation between the primed and the unprimed location for the two distances. The presence of a line did not change this result.

To summarize, the PSS results do not support the notion of a graded focus of attention. Experiment 2 was designed to test if this pattern of results was reliable. We further tried to optimise the experimental conditions such that the probability of finding an attentional gradient increased. We reduced the distance between the two targets in both conditions. Some ILM experiments revealed gradients of attention when lines of 5 to $10^{\circ}$, or even longer lines, were used (e.g., Hikosaka et al., 1993a, b, c). However, it is not clear from these results whether the gradient or the illusory line motion encompasses the whole line or only parts of it, that is, whether line length is a measure of gradient size. Also, some gradients of attention were much smaller in diameter (e.g., LaBerge, 1983). Thus, it is possible that both of the distances compared in Experiment 1 fell outside the gradient. Consequently, Experiment 2 compares much smaller distances.

Note also that although we did not reject the null hypothesis that latency priming is the same for the small and the large distance, that is, $5.4^{\circ}$ and $9.1^{\circ}$ between the focus of attention and the unprimed target, latency facilitation was numerically $11 \mathrm{~ms}$ larger in the near/no line condition than in all of the other
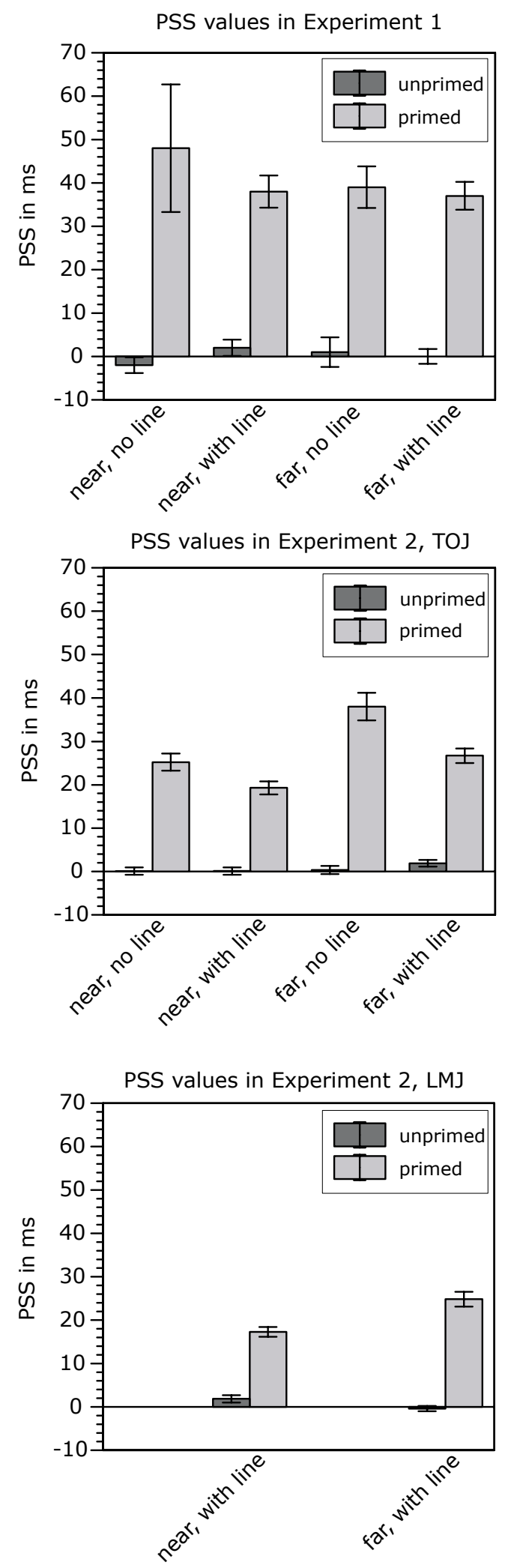

Figure 2.

PSS values in Experiment 1 (top panel) and in Experiment 2 (centre panel: TOJ task; bottom panel: LMJ task). The error bars indicate standard errors of the mean, that is, between-subjects variability. In Experiment 1, no gradient effect is visible; in Experiment 2, line presence as well as distance influence the PSS in primed conditions. 
conditions. The reason for this finding is unclear considering that the standard error was heavily increased in this condition (15 as compared to approximately $4 \mathrm{~ms}$; compare Figure 2, top panel).

\section{EXPERIMENT 2}

Experiment 2 is identical to Experiment 1 , except that target distances were reduced to $2.4^{\circ}$ and $1.5^{\circ}$ in the far and near conditions, respectively. Also, we included a session in which the participants judged the direction of motion within the line (line-motion judgment; LMJ). Using this method, we attempted a comparison between two tasks under the same stimulus conditions.

The experimental rationale in the LMJ task was basically the same as in the TOJ task. If, in unprimed conditions, the first target captures attention and causes an attentional gradient, the line should develop gradedly and ILM should be visible. If, in primed conditions, the prime captures attention, the gradient should be centered around its location and the probability that line motion is perceived away from this location should increase. Facilitation was calculated in exactly the same way as in the TOJ task: From the LMJs, we estimated the target SOA at which the participants equally often perceived motion in one or the other direction (PSS). The amount of attentional facilitation was again calculated as the difference between the PSS of unprimed and the PSS of primed conditions.

\section{Method}

\section{Participants}

Twenty women and 4 men (mean age 25 years) who had not taken part in Experiment 1 and whose informed consent was obtained received $€ 10$ for participation. Four participants did not show up for the second session, and their data from the first session were discarded.

\section{Apparatus and stimuli}

The apparatus and stimuli were identical to those used in Experiment 1, except that the distance between the targets was 2.4 or $1.5^{\circ}$ of visual angle. In order to avoid crowded displays, we increased the resolution of the computer screen to $1024 \times 768$ pixels. This reduced the edge length of the targets to $0.9^{\circ}$, that of the prime to $0.3^{\circ}$, and line length to 1.4 or $0.5^{\circ}$.

Further, we included an LMJ session. In this session, only trials with the line were presented. It contained
640 trials in random order; the TOJ session consisted of a total of 1,280 trials.

\section{Procedure}

The procedure was the same as in Experiment 1, except for the following: The participants worked through a TOJ and an LMJ task in random order on different days. In the LMJ session, they judged the direction in which the line seemed to unfold. The instruction was as parallel to the TOJ as possible. The participants judged the direction of motion with a ternary judgment including two order categories ("motion towards red target" vs. "motion towards blue target") and a simultaneous/doubt category ("no motion/unclear"). Half of the participants responded with the left mouse button if they saw the line shoot towards the red target, and with the right button if they perceived the line as shooting towards the blue target. The assignment was reversed for the other half. The center button could be used to indicate simultaneity or uncertainty.

\section{Results}

In a first step, we checked whether the order of the tasks influenced the results. Our reasoning in this experiment rests upon the assumptions that the participants perceive a temporal order of the targets and motion within the line, and that these impressions are systematically changed by the presence of the prime: The primed target is more often perceived as the first one than the unprimed target, and line motion more often begins at the location of the primed than at the location of the unprimed target. However, it is also possible that either our displays did not convey a line motion or that latency facilitation by a masked prime does not show up in LMJs, but - importantly - that the observers transfer their judgment strategy from the TOJ to the LMJ. In the latter case, observers who begin with the LMJ task, and thus cannot transfer the judgment strategy from the TOJ task, should differ from observers who begin with the TOJ task.

To check for the presence of such an influence, we performed repeated-measures ANOVAs including task order as a between-subjects factor, separately for the TOJ task and the LMJ task. Order of tasks did not reach significance as a main effect and did not enter into a significant interaction (all $F \leq 2.1$, all $p s \geq .19$ ). Although we cannot definitely exclude a strategy transfer on the basis of not rejecting the null hypothesis, its effect (if present) is at the most only minor. We thus abandoned the group factor in the following analyses. 


\section{TOJ task}

A three-way ANOVA of PSS revealed a main effect of priming $[F(1,19)=239.08, p<.001]$. The main effects of distance and line presence were also significant [distance: $F(1,19)=42.35, p<.001$; line presence: $F(1,19)=21.13, p<.001]$. Priming and Line presence as well as Priming and Distance interacted $[F(1,19)=55.6, p<.001, F(1,19)=36.99$, $p<.001$, respectively]. These interactions can easily be explained: PSS was Zero in all of the unprimed conditions, and it was independent of whether there was a line in the display and how large the distance between the stimuli was. By contrast, the PSS values in the primed conditions depended both on line presence and distance. That is, the influence of the prime, rather than temporal order perception, was mediated by the distance of the second target and by the line which connected the attended location to this second target. There was, however, no significant interaction of Line presence and Distance $[F(1,19)=1.71$, $p=.21]$, and the three-way interaction just failed to reach significance $[F(1,19)=3.17, p=.09]$. PLP was reduced by $9 \mathrm{~ms}$ both by the presence of a line and by the shorter distance.

PSS values were on average $+1 \mathrm{~ms}$ in unprimed conditions, and ranged from $38 \mathrm{~ms}$ in the condition without the line and with a large distance between stimuli to $19 \mathrm{~ms}$ in the condition with the line and short distance between stimuli (compare Figure 2, centre panel).

\section{LMJ task}

Analogous to the TOJ task, the PSS in the LMJ relates to perceived simultaneity. The PSS value indicates the SOA between the targets at which no motion (or motion with an unclear direction) is perceived in the line. If the PSS deviates from zero, this indicates that no motion is perceived even though the targets appear asynchronously. This is again quite analogous to the TOJ task, in which a PSS value which differs from zero indicates that the targets appear as simultaneous even though they are in fact asynchronous.

In the PSS data, there was a main effect of priming $[F(1,19)=209.13, p<.001]$, a main effect of distance $[F(1,19)=6.76, p<.05]$, and a significant interaction of both factors $[F(1,19)=29.16, p<.001]$. Again, the shorter distance reduced priming by $10 \mathrm{~ms}$.

PSS values were on average $1 \mathrm{~ms}$ in the unprimed conditions, $25 \mathrm{~ms}$ with large distance and $17 \mathrm{~ms}$ with short distance; PLP was 25 in the former and $15 \mathrm{~ms}$ in the latter condition (compare Figure 2, bottom panel).

\section{Comparison of TOJ and $L M J$}

We compared TOJ and LMJ data in an additional ANOVA; the TOJ data of the conditions without prime were omitted, because these conditions could not be realised in the LMJ task. According to this analysis, there is no statistical difference between these two tasks $[F(1,19)=2.2$, $p=.15]$, and task does not interact with either distance $[F(1,19)=3.19, p=.09]$, or priming $[F(1,19)=1.93, p=.18]$, or both $[F(1,19)=3.31$, $p=.09]$. However, some of these comparisons are close to significance, and the power is too small to allow a conclusion to be drawn about the difference between the TOJ and LMJ tasks (given a medium effect size and an a level of .05, power is .376). Figure 2 (bottom panel) indicates that priming is slightly less effective in the LMJ when the distance is small (4 ms). As expected, the main effects of priming and distance and the interaction between Distance and Priming again reached significance [priming: $F(1,19)=259.92$, $p<.001$; distance $F(1,19)=18.7, p<.001$; interaction: $F(1,19)=23.09, p<.001]$.

All of the PLP values differed significantly from zero, both in the TOJ and the LMJ task (independent t-Tests; all $t s \geq 6.97$, all $p s \leq .001)$.

\section{Discussion}

The results of Experiment 2 differ substantially from Experiment 1. Although PLP was present in all of the primed conditions, its size varied considerably between the conditions: We found that the presence of a line reduced PLP by $9 \mathrm{~ms}$ in the TOJ task. This reduction might be due to the line facilitating the spread of attention from the primed to the unprimed location. However, other explanations are conceivable. For example, the line might have induced more perceptual grouping between the targets, and thereby have absorbed part of the priming effect.

In the LMJ task, the effect of line presence could not be tested because only conditions with line were included. Reducing the distance between the attended and the unattended location from $2.4^{\circ}$ to $1.5^{\circ}$ reduced the priming effect by $9 \mathrm{~ms}$ in the TOJ task and by $10 \mathrm{~ms}$ in the LMJ task. Thus, we found evidence for a gradient of attention within $2.5^{\circ}$.

Also, we compared LMJ and TOJ directly under the same stimulus conditions. Similar to an earlier study (Scharlau, 2004b), we found PLP in both tasks, and its size did not differ largely (and, as yet, statistically). This supports the claim that TOJ and LMJ measure a 
similar kind of attention-mediated facilitation, even though the perceptual measures concern different phenomenal and functional aspects, that is, temporal order in the TOJ and motion perception in the LMJ.

\section{GENERAL DISCUSSION}

The present study investigated whether the largely indirect evidence for attentional gradients provided by ILM generalizes to a functionally closely related phenomenon of attentional facilitation, perceptual latency priming, in which gradients can be directly measured. With distances larger than $5^{\circ}$ of visual angle, evidence for gradients was weak. Statistically, the degree of perceptual latency facilitation was independent of the distance between the targets. It was furthermore independent of the presence of a line, as well as the combined effects of line presence and distance, which is the critical test for the assumption that attentional gradients require the presence of stimulation (Hamm \& Klein, 2002; Zimba \& Hughes, 1987).

The picture changed when smaller distances (as well as smaller stimuli) were used in Experiment 2. Attention-mediated latency facilitation was present, but it was diminished both by the presence of a line and by reduced distance between the targets. The latter finding is in accord with the gradient account.

How can the PSS results be explained? We find a difference between a distance of $2.4^{\circ}$ and a distance of $1.5^{\circ}$ (Experiment 2), but not with larger distances (Experiment 1). This finding conforms best with a small $\left(<5^{\circ}\right)$ focus of attention within which facilitation gradually declines.

Note, however, that PLP was smaller in the present study than in earlier investigations (e.g., Scharlau \& Neumann, 2003b). This may indicate that there was some leakage of facilitation from the primed location to the location of the unprimed target even in the conditions with large distances in Experiment 1. Remember that the presence of PLP indicates that latency facilitation is larger - that is, attention stronger - at the primed than at the unprimed location, but that this measure cannot be used to show that there is no facilitation at all at the unprimed location. Thus, it is possible that some attentional facilitation spread towards the unprimed location in all of the distance conditions of Experiments 1 and 2 . This leakage does not, however, vary with distances between about 5 and $9^{\circ}$ of visual angle. Note also that the precise shape of the attentional gradient cannot be inferred from the present results. They are compatible with a monotonously decreasing attentional gradient as well as more complex shapes, such as, for instance, a Mexican hat, that is, a region of inhibition around the attentional focus (Müller, Mollenhauer, Rösler, \& Kleinschmidt, 2005). For a test of the precise shape, more distances need to be tested.

Earlier findings show that PLP may also depend on spatial distance if the distance between prime and primed target is varied. If the targets in this situation are sustained (i.e., deleted only after the judgment was made), PLP shows a graded distribution, declining to Zero within at most $2.5^{\circ}$ (Scharlau, 2004c). This agrees well with the present findings, as well as with other studies in which a gradient of attention with a small diameter was found (e.g., LaBerge, 1983).

Consequently, the present results lend weight to doubts about the attentional-gradient explanation of ILM (see Downing \& Treisman, 1997; see also Jancke, Chavane, Naaman, \& Grinvald, 2004, for a non-attentional account of ILM). In light of the present findings, attentional facilitation can account for perceived motion of the line, but only for line parts very close to the attended or primed location. Motion in a wider spatial range - if present, remember that this is rarely directly assessed in ILM studies - must have a different functional basis. Future research should directly investigate, for instance, whether and how far classic apparent motion and response bias contribute to ILM. Besides, the results add to the evidence that attention is not necessarily distributed in a gradient fashion (e.g., Downing, 1988; Goolkasian \& Tarantino, 1999; Handy et al., 1996; Hodgson et al., 1999; Hughes \& Zimba, 1985, 1987).

Further, we attempted to compare TOJs and LMJs under exactly the same stimulus conditions. Similar to a former study (Scharlau, 2004b), the results were essentially the same for both measures, that is, PLP can be assessed both with a temporal order judgment and on the basis of illusory perception of motion within a line. Its size also does not differ largely across these measures. These findings thus backup the claim that both phenomena reflect attention-mediated facilitation. This finding might also support the conjecture that attention-mediated line motion is confined to a few degrees of visual angle around the prime and that the impression of motion in other parts of the line might have a different functional basis (see above). Also, whether these results transfer to ILM caused by a visual cue remains at present an open question.

Finally, the present experiments demonstrate that the masked-priming paradigm may be used as a means for studying the processing of information. Numerous studies on sensorimotor processing of masked information have been conducted in the last decade (e.g., Ansorge, 2004; Ansorge \& Neumann, 
2005; Jaśkowski, Skalska, \& Verleger, 2003; Klotz \& Neumann, 1999; Lleras \& Enns, 2004; Schmidt, 2002; Verleger, Jaśkowski, Aydemir, van der Lubbe, \& Groen, 2004; Vorberg, Mattler, Heinecke, Schmidt, \& Schwarzbach, 2003, see also Kiesel, Kunde, Pohl, \& Hoffmann, 2006, this volume; Schlaghecken \& Sisman, 2006, this volume). On the other hand, temporal order judgments and prior entry in general have been shown to be useful for the investigation of a multitude of attention-related topics, such as the role of subcortical attentional processing (Zackon et al., 1999), attentional deficits in extinction (Rorden, Mattingley, Karnath, \& Driver, 1997; see also Karnath, Zimmer, \& Lewald, 2002), or unilateral neglect (Robertson, Mattingley, Rorden, \& Driver, 1998). The present study, which concerns the attentional processing of masked inofmration, contributes to the small body of studies on the intersection of these topics (e.g., Jaśkowski, van der Lubbe, Schlotterbeck, \& Verleger, 2002; McCormick, 1997; Scharlau \& Ansorge, 2003). Note, however, that although the displays used in the present experiments met the conditions for metacontrast masking, we did not assess masking strength. As yet, no definite conclusions about ILM by perfectly masked stimuli can be drawn form the present data.

In recent years, the question whether attention can be controlled by masked information has gained more and more interest. For example, Ansorge and Heumann (2006, this volume) and Skalska, Jaśkowski, and van der Lubbe (2006, this volume) used speeded choice responses and electrophysiological correlates of attentional selection to investigate the degree to which the control of attention by masked primes is mediated by top-down influences such as current intentions versus bottom-up processes such as stimulus-driven capture. The present experiments pursue the impact of masked information on attention on the perceptual level.

\section{Author note}

Ingrid Scharlau and Gernot Horstmann, Department of Psychology, Bielefeld University, Germany.

Supported by grant $\mathrm{Ne} 366 / 5-3$ of the Deutsche Forschungsgemeinschaft to Odmar Neumann. We would like to thank Ulrich Ansorge and two anonymous reviewers for helpful suggestions, and Lily-Maria Silny and Heike Hartwig-Jakobs for help with preparing the manuscript.

\section{References}

Ansorge, U. (2004). Top-down contingencies of nonconscious priming revealed by dual-task interfer- ence. Quarterly Journal of Experimental Psychology, 57A, 1123-1148. |Www|

Ansorge, U., \& Heumann, M. (2006). Shifts of visuospatial attention to invisible (metacontrast-masked) singletons: Clues from reaction times and event-related potentials. Advances in Cognitive Psychology, 2, 61-76. ACP

Ansorge, U., \& Neumann, O. (2005). Intentions determine the effect of invisible metacontrast-masked primes: Evidence for top-down contingencies in a peripheral cueing task. Journal of Experimental Psychology: Human Perception and Performance, 31, 762-777.

Bessel, F. W. (1838). Untersuchungen über die Wahrscheinlichkeit der Beobachtungsfehler (Studies on the probability of observational errors). Astronomische Nachrichten (Astronomical News), 15, 369-404.

Boring, E. G. (1929). A history of experimental psychology. New York: Appleton-Century.

Downing, C. J. (1988). Expectancy and visual-spatial attention: Effects on perceptual quality. Journal of Experimental Psychology: Human Perception and Performance, 14, 188-202.

Downing, P., \& Treisman, A. (1997). The line-motion illusion: Attention or impletion? Journal of Experimental Psychology: Human Perception and Performance, 23, 768-779.

Eriksen, C. W., \& Hoffman, J. E. (1972). Temporal and spatial characteristics of selective encoding from visual displays. Perception \& Psychophysics, 12, 201-204.

Faul, F., \& Erdfelder, E. (1992). GPOWER: A prior, posthoc, and compromise power analyses for MS-DOS [Computer program]. Bonn, FRG: Bonn University, Dep. of Psychology.

Finney, D. J. (1971). Probit analysis (3rd ed.). Cambridge: University Press.

Goolkasian, P. A., \& Tarantino, M. (1999). Covert and overt attention and the processing of cues for location and target identification. Journal of General Psychology, 126, 235-260.

Grünau, M. V., Racette, L., \& Kwas, M. (1996). Measuring the attentional speed-up in the motion induction effect. Vision Research, 36, 2433-2446. Www Grünau, M. V., Saikali, Z., \& Faubert, J. (1995). Processing speed in the motion induction effect. Perception, 24, 477-490. www

Hamm, J. P., \& Klein, R. M. (2002). Does attention follow the motion in the "shooting line" illusion? Perception \& Psychophysics, 64, 279-291. WWW

Handy, T. C., Kingstone, A., \& Mangun, G. R. (1996). Spatial distribution of visual attention: Perceptual 
sensitivity and response latency. Perception \& Psychophysics, 58, 613-627. |WWw

Hawkins, H. L., Hillyard, S. A., Luck, S. J., \& Mouloua, M. (1990). Visual attention modulates signal detectability. Journal of Experimental Psychology: Human Perception and Performance, 16, 802-811. WwW

Henderson, J. M., \& Macquistan, A. D. (1993). The spatial distribution of attention following an exogenous cue. Perception \& Psychophysics, 53, 221-230. |www|

Hikosaka, O., Miyauchi, S., \& Shimojo, S. (1993a). Focal visual attention produces illusory temporal order and motion sensation. Vision Research, 45, 1219-1240. $\underline{\underline{W W} \mid}$

Hikosaka, O., Miyauchi, S., \& Shimojo, S. (1993b). Visual attention revealed by an illusion of motion. Neuroscience Research, 18, 11-18. Www

Hikosaka, O., Miyauchi, S., \& Shimojo, S. (1993c). Voluntary and stimulus-induced attention detected as motion sensation. Perception, 22, 517-526. Www

Hodgson, T. L., Müller, H. J., \& O'Leary, M. J. (1999). Attentional localization prior to simple and directed manual responses. Perception \& Psychophysics, 61, 308-321. WwW

Hughes, H. C., \& Zimba, L. D. (1985). Spatial maps of directed visual attention. Journal of Experimental Psychology: Human Perception and Performance, 11, 409-430. Www

Hughes, H. C., \& Zimba, L. D. (1987). Natural boundaries for the spatial spread of directed visual attention. Neuropsychologia, 25, 5-18. Www

Jancke, D., Chavane, F., Naaman, S., \& Grinvald, A. (2004). Imaging cortical correlates of illusion in early visual cortex. Nature, 428, 423-426. |www

Jaśkowski, P., Skalska, B., \& Verleger, R. (2003). How the self controls its "automatic pilot" when processing subliminal information. Journal of Cognitive Neuroscience, 15, 911-920. WwW

Jaśkowski, P., van der Lubbe, R. H. J., Schlotterbeck, E., \& Verleger, R. (2002). Traces left on visual selective attention by stimuli that are not consciously identified. Psychological Science, 13, 48-54. WwW

Karnath, H. O., Zimmer, U., \& Lewald, J. (2002). Impaired perception of temporal order in auditory extinction. Neuropsychologia, 40, 1977-1982. www

Kiesel, A., Kunde, W., Pohl, C., \& Hoffmann, J. (2006). Priming for novel masked stimuli depends on target set size. Advances in Cognitive Psychology, 2, 37-45. ACP

Klotz, W., \& Neumann, O. (1999). Motor activation without conscious discrimination in metacontrast masking. Journal of Experimental Psychology: Human Perception and Performance, 25, 976-
$-992$.

LaBerge, D. (1983). Spatial extent of attention to letters and words. Journal of Experimental Psychology: Human Perception and Performance, 9, 371-379. |Www Lleras, A., \& Enns, J. (2004). Negative compatibility or object updating? A cautionary tale of mask-dependent priming. Journal of Experimental Psychology: General, 133, 475-493. WwW

Mangun, G. R., \& Hillyard, S. A. (1988). Spatial gradients of visual attention: Behavioral and electrophysiological evidence. Electroencephalography and clinical Neurophysiology, 70, 417-428. |Www

McCormick, P. A. (1997). Orienting attention without awareness. Journal of Experimental Psychology: Human Perception and Performance, 23, 168-180. Www McCormick, P. A., \& Klein, R. (1990). The spatial distribution of attention during covert visual orienting. Acta Psychologica, 75, 225-242. |WWW

Müller, H. J., \& Findlay, J. M. (1987). Sensitivity and criterion effects in the spatial cuing of visual attention. Perception \& Psychophysics, 42, 383-399. WwW

Müller N. G., Mollenhauer, M., Rösler, A., \& Kleinschmidt, A. (2005). The attentional field has a Mexican hat distribution. Vision Research 45, 1129-1137. [WwW

Neumann, O. (1982). Experimente zum Fehrer-RaabEffekt und das "Wetterwart"-Modell der visuellen Maskierung. [Experiments on the Fehrer-Raab effect and the "Weather Station" Model of visual masking.] Report No. 24/1982, Department of Psychology at the Ruhr-University of Bochum, Cognitive Psychology Unit.

Neumann, O., Esselmann, U., \& Klotz, W. (1993). Differential effects of visual-spatial attention on response latency and temporal order judgment. Psychological Research, 56, 26-34. |Www

Pashler, H. (1998). The psychology of attention. Cambridge, MA: MIT Press.

Posner, M. I. (1980). Orienting of attention. Quarterly Journal of Experimental Psychology, 32, 3-25. |WWW

Robertson, I. H., Mattingley, J. B., Rorden, C., \& Driver, J. (1998). Phasic alerting of neglect patients overcomes their spatial deficit in visual awareness. Nature, 395, 169-173.

Rorden, C., Mattingley, J. B., Karnath, H.-O., \& Driver, J. (1997). Visual extinction and prior entry: Impaired perception of temporal order with intact motion perception after unilateral parietal damage. Neuropsychologica, 35, 421-433. WwW

Sanford, E. C. (1888). Personal equation. The American Journal of Psychology, 2, 3-38, 271-298, 403-430.

Scharlau, I. (2002). Leading, but not trailing, primes 
influence temporal order perception: Further evidence for an attentional account of perceptual latency priming. Perception \& Psychophysics, 64, 1346-1360.

Scharlau, I. (2004a). Evidence against a response bias in temporal order judgments with attention manipulation by masked primes. Psychological Research, 68, 224-236.

Scharlau, I. (2004b). Illusory line motion and perceptual latency priming: Two alternative measures for attentional facilitation. In R. P. Würtz, \& M. Lappe (Eds.), Dynamic Perception 2004: Workshop of GI section 1.0.4 "Image Understanding" and the European Networks MUHCI and ECOVISION; Tübingen University, November 2004 (127-133). Berlin, Amsterdam: AKA IOS Press.

Scharlau, I. (2004c). Spatial distribution of visual attention in perceptual latency priming. Quarterly Journal of Experimental Psychology, 57A, 1411-1437. Www

Scharlau, I. (2005). Perceptual latency priming: A measure of attentional facilitation. Manuscript submitted for publication.

Scharlau, I., \& Ansorge, U. (2003). Direct parameter specification of an attention shift: Evidence from perceptual latency priming. Vision Research, 43, 1351-1363. $\mid \mathrm{wWw}$

Scharlau, I., \& Neumann, O. (2003a). Perceptual latency priming by masked and unmasked stimuli: Evidence for an attentional explanation. Psychological Research, 67, 184-197. www

Scharlau, I., \& Neumann, O. (2003b). Temporal parameters and time course of perceptual latency priming. Acta Psychologica, 113, 185-203. Www

Schlaghecken, F., \& Sisman, R. (2006). Intact lowlevel motor inhibition in children: Evidence from the negative compatibility effect. Advances in Cognitive Psychology, 2, 7-19. ACP

Schmidt, T. (2002). The finger in flight: Real-time motor control by visually masked color stimuli. Psychological Science, 13, 112-117. Www

Shimojo, S., Hikosaka, O., \& Miyauchi, S. (1999). Automatic and controlled attention detected by the line motion effect. In D. Gopher \& A. Koriat (Eds.), Attention and performance XVII (pp. 145-163). Cambridge, MA: The MIT Press.

Shore, D. I., Spence, C., \& Klein, R. M. (2001). Visual prior entry. Psychological Science, 12, 205-212. WWW

Skalska, B., Jaśkowski, P., \& van der Lubbe, R. (2006). Direct parameter specification and visuo-spatial attention in subliminal priming of motor reaction Advances in Cognitive Psychology, 2, 47-59. ACP

Steglich, C., \& Neumann, O. (2000). Temporal, but not spatial, context modulates a masked prime's effect on temporal order judgment, but not on response latency. Psychological Research, 63, 36-47. WWW

Stelmach, L. B., Campsall, J. M., \& Herdman, C. M. (1997). Attentional and ocular movements. Journal of Experimental Psychology: Human Perception and Performance, 23, 823-844.

Stelmach, L. B., \& Herdman, C. M. (1991). Directed attention and perception of temporal order. Journal of Experimental Psychology: Human Perception and Performance, 17, 539-550. Www

Titchener, E. M. (1908). Lectures on the elementary psychology of feeling and attention. New York: MacMillan.

Treisman, A. M. (1988). Features and objects: The Fourteenth Bartlett Memorial Lecture. Quarterly Journal of Experimental Psychology, 40A, 201-237.

Turatto, M., \& Galfano, G. (2001). Attentional capture by color without any relevant attentional set. Perception \& Psychophysics, 63, 286-297. |www

Ulrich, R. (1987). Threshold models of temporal order judgments evaluated by a ternary response task. Perception \& Psychophysics, 42, 224-239. |www

Verleger, R., Jaśkowski, P., Aydemir, A., van der Lubbe, R., \& Groen, M. (2004). Qualitative differences between conscious and nonconscious processing? On inverse priming induced by masked arrows. Journal of Experimental Psychology: General, 133, 494-515. $\underline{w w}$

Vorberg, D., Mattler, U., Heinecke, A., Schmidt, T., \& Schwarzbach, J. (2003). Different time courses for visual perception and action priming. Proceedings of the National Academy of Sciences (USA), 100, 6275-6280. WwW

Wundt, W. (1887). Grundzüge der physiologischen Psychologie (Main features of physiological psychology). Leipzig: Engelmann.

Zackon, D. H., Casson, E. J., Zafar, A., Stelmach, L., \& Racette, L. (1999). The temporal order judgment paradigm: Subcortical attentional contribution under exogenous and endogenous cueing conditions. Neuropsychologia, 37, 511-520. |WWw

Zimba, L. D., \& Hughes, H. C. (1987). Distractor-target interactions during directed visual attention. Spatial Vision, 2, 117-149. Www 